\title{
THE BISTABLE POTENTIAL: AN ARCHETYPE FOR CLASSICAL AND QUANTUM SYSTEMS
}

\author{
B. SPAGNOLO*,†, P. CALDARA, A. LA COGNATA and D. VALENTI ${ }^{\ddagger}$ \\ Dipartimento di Fisica, Università di Palermo, \\ Group of Interdisciplinary Physics and CNISM, \\ Viale delle Scienze, Edificio 18, I-90128 Palermo, Italy \\ †bernardo.spagnolo@unipa.it \\ $\ddagger$ davide.valenti@unipa.it
}

\section{A. FIASCONARO}

Departamento de Física de la Materia Condensada ICMA (CSIC-Universidad de Zaragoza), 50009 Zaragoza, Spain

afiascon@unizar.es

\section{A. A. DUBKOV}

Radiophysics Department, Nizhny Novgorod State University, 23 Gagarin Avenue, 603950 Nizhny Novgorod, Russia

dubkov@rf.unn.ru

\section{G. FALCI}

Dipartimento di Fisica e Astronomia, Università di Catania, Via Santa Sofia, 64, I-95123 Catania, Italy gfalci@dmfci.unict.it

In this work we analyze the transient dynamics of three different classical and quantum systems. First, we consider a classical Brownian particle moving in an asymmetric bistable potential, subject to a multiplicative and additive noise source. We investigate the role of these two noise sources on the life time of the metastable state. A nonmonotonic behavior of the lifetime as a function of both additive and multiplicative noise intensities is found, revealing the phenomenon of noise enhanced stability. Afterward, by using a Lotka-Volterra model, the dynamics of two competing species in the presence of Lévy noise sources is analyzed. Quasiperiodic oscillations and stochastic resonance phenomenon in the dynamics of the competing species are found. Finally the dynamics of a quantum particle subject to an asymmetric bistable potential and interacting with a thermal reservoir is investigated. We use the Caldeira-Leggett model and the approach

*Invited talk at the International Conference on Statistical Physics, 11-15 July 2011, Cyprus. 
of the Feynman-Vernon functional in discrete variable representation. We obtain the time evolution of the population distributions in energy eigenstates of the particle, for different values of the coupling strength with the thermal bath.

Keywords: Metastability and bistability; multiplicative noise; noise enhanced stability; stochastic resonance; population dynamics; open quantum systems.

\section{Introduction}

Relaxation phenomena in many natural systems proceed through metastable states, often observed in condensed matter physics, and also in various other fields, from cosmology to biology and high-energy physics. ${ }^{1-4}$ In this work we analyze relaxation phenomena in three different classical and quantum systems. (i) First, we consider a classical system with asymmetric bistable potential, and investigate the role of a multiplicative and an additive noise source on the life time of the metastable state. A nonmonotonic behavior of the lifetime as a function of both additive and multiplicative noise source intensities is found, revealing the phenomenon of noise enhanced stability (NES). ${ }^{5-7}$ (ii) In the second system the dynamics of two competing species, in the presence of alpha stable Lévy noise sources, ${ }^{8}$ is analyzed. By using a Lotka-Volterra model we study the time behavior of this ecosystem interacting with the noise sources, which mimic the surrounding environment. The role of the two non-Gaussian noise sources in the exclusion and coexistence regimes is analyzed, and stochastic resonance phenomenon in the dynamics of the competing species is found. (iii) Finally, the dynamics of a quantum particle subject to an asymmetric bistable potential and interacting with a thermal reservoir, that is the environmental noise, is investigated. We use the Caldeira-Leggett model and the approach of the Feynman-Vernon functional in discrete variable representation (DVR). ${ }^{9}$ We obtain the time evolution of the population distributions in energy eigenstates of the particle, varying the value of the coupling strength between particle and thermal bath.

\section{Metastable State with Multiplicative Noise}

Systems described by equations, whose noise amplitude is proportional or in general is a function of the order parameter, are ubiquitous in many different areas of scientific investigation. Such equations are said multiplicative Langevin equations (MLE) because the noise amplitude depends on the state variables themselves. Systems exhibiting absorbing states and noise-induced phase transitions can be described by these equations. ${ }^{10-14}$ The models based on MLE abound not only in physics, but also in biology, ecology, economy, or social sciences. The function of the order parameter $g(x)$ takes different forms depending on the system investigated, ranging from a pure linear multiplicative noise term, often used in population dynamics, to more general expressions which represent the effects of both additive and multiplicative noise sources. ${ }^{15,16}$ 
Recently, metastability and nucleation in a kinetic two-dimensional Ising model has been analyzed using a Langevin equation with additive and multiplicative noise sources. The nonlinear interplay between the thermal fluctuations, that is the additive noise, and the nonequilibrium fluctuations, that is the multiplicative noise, gives rise to the noise enhanced stabilization of the metastable state. ${ }^{17}$

Here we focus on the effect of both the additive and the multiplicative noise on a Brownian particle moving in an asymmetric bistable potential, characterized by a metastable state. We investigate the average life time of the metastable state for the Brownian particle starting from an initial unstable state. Using functional analysis we obtain the Fokker-Planck equation, corresponding to the Langevin multiplicative equation, and the stationary probability density function (PDF). The system shows the presence of an increase of stability as a function of both noise intensities (multiplicative and additive), giving rise to a nonmonotonic behavior of the average life time of the metastable state. This confirms that a suitable noise intensity can stabilize metastable systems. ${ }^{18,19}$

\subsection{The Model}

The Langevin equation of a Brownian particle driven by a noise source is given by the following MLE

$$
\frac{d x(t)}{d t}=f(x(t))+g(x(t)) \xi(t),
$$

where $f(x(t))$ and $g(x(t))$ are arbitrary deterministic functions of the order parameter $x(t)$, and $\xi(t)$ is a random force which in our case is a Gaussian white noise with the usual statistical properties: $\langle\xi(t)\rangle=0$ and $\langle\xi(t) \xi(t+\tau)\rangle=2 \mathcal{D} \delta(\tau)$. Using Eq. (1), the relation $P(x, t)=\langle\delta(x-x(t))\rangle$ and the ordinary calculus we obtain the corresponding Fokker-Planck equation for the $\mathrm{PDF}^{21,22}$

$$
\frac{\partial P(x, t)}{\partial t}=-\frac{\partial}{\partial x}[f(x) P(x, t)]+\mathcal{D} \frac{\partial}{\partial x}\left[g(x) \frac{\partial}{\partial x}[g(x) P(x, t)]\right] .
$$

This is the "Stratonovich form" of the Fokker-Planck equation, because we used the ordinary calculus. The asymptotic stationary distribution is obtained from Eq. (2) by putting $\left(\partial P_{\text {st }}(x, t) / \partial t\right)=0$, that is

$$
P_{\mathrm{st}}(x)=\frac{\mathcal{N}}{g(x)} \exp \left(-\int \frac{f\left(x^{\prime}\right)}{\mathcal{D} g^{2}\left(x^{\prime}\right)} d x^{\prime}\right)=\mathcal{N} e^{-\frac{V_{\text {eff }}(x)}{D}},
$$

where the value of $\mathcal{N}$ is given by the normalization condition. From Eq. (3) we see that the positions of the minima and maxima are depending on the functional form of $g(x) .{ }^{10}$ We choose for our model $g(x)=\sqrt{D+\mu x^{2}}$, where $D$ is the strength of the additive noise, i.e., thermal noise, and $\mu$ is the intensity of the multiplicative noise. The deterministic driving force is $f(x)=-(d V / d x)$, and $V(x)=4 x^{4}+2 x^{3}-8 x^{2}-6 x$ is an asymmetric quartic potential (see Fig. 1), with two minima at $x=1$ and $x=-1$ (metastable state), and a maximum at $x=-3 / 8$. In Fig. 1 we show: (i) the 


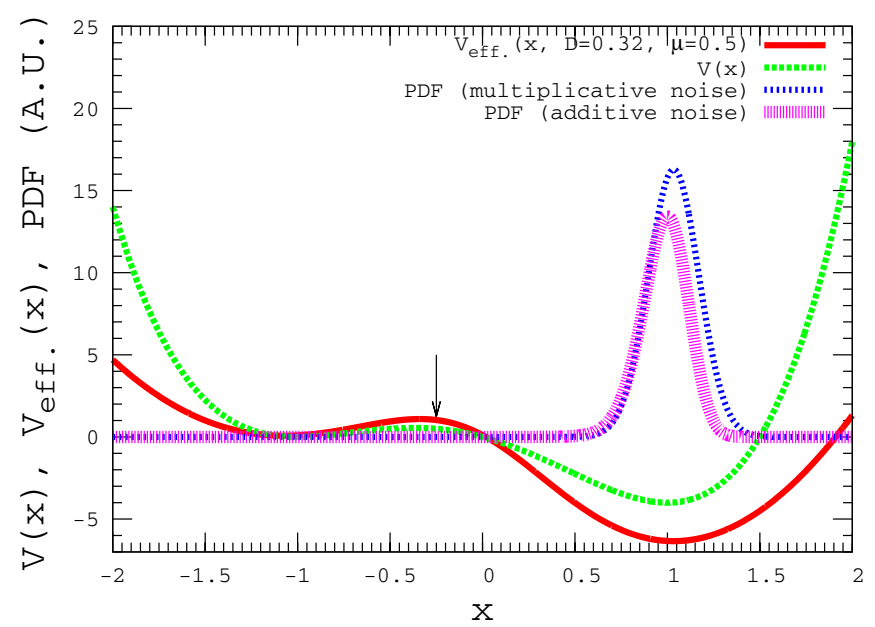

Fig. 1. (Color online) The potential $V(x)$ (green dot line) and the corresponding PDF (magenta dot line) for the pure additive noise case $(D=0.32$ and $\mu=0.0)$. The effective potential $V_{\text {eff }}$ (continuous red line) and the corresponding PDF (blue dot line) in the presence both of multiplicative and additive noise sources $D=0.32$ and $\mu=0.5$. The arrow indicates the initial position of the Brownian particle.

potential $V(x)$ and the PDF for the pure additive noise case, (ii) the effective potential $V_{\text {eff }}$ and the corresponding PDF in the presence both of multiplicative and additive noise sources with intensities respectively $\mu=5$ and $D=0.32$.

\subsection{Simulation results}

In order to explore the role played by parameters $D$ and $\mu$, we calculated the numerical solutions of Eq. (1), obtaining the mean escape time (MET). We performed our simulations by choosing for the system the initial unstable condition $x=-0.25$, just on the right of the maximum (see Fig. 1), and an absorbing barrier at $x=0.99$. The results show that there is a nonmonotonicity of the MET in both the parameters $D$ and $\mu$. In Fig. 2 we show the behavior of the MET as a function of the parameter $D$ for five different values of the parameter $\mu$, namely $\mu=0.0,0.04,0.15,0.19,0.23$. We observe that for every value of $\mu$ we have the same nonmonotonic trend with a maximum. In particular, for increasing values of $\mu$ the maximum of the MET decreases and shifts toward low values of the parameter $D$. In Fig. 3 we show the behavior of MET versus the multiplicative noise intensity $\mu$ for different values of $D$, namely $D=0.0,0.04,0.08,0.12,0.16$.

The NES effect is almost absent in the presence of a pure multiplicative noise source $(D=0.0)$. We observe that increasing of the value of parameter $D$ up to $\sim 0.2$ causes an enhancement of the NES effect. Simulations performed for higher values of $D$, not reported here, show that the maximum of MET decreases considerably. This means that there is an optimum range of values both of multiplicative and additive noise intensities for which the enhancement of metastability is observed. 


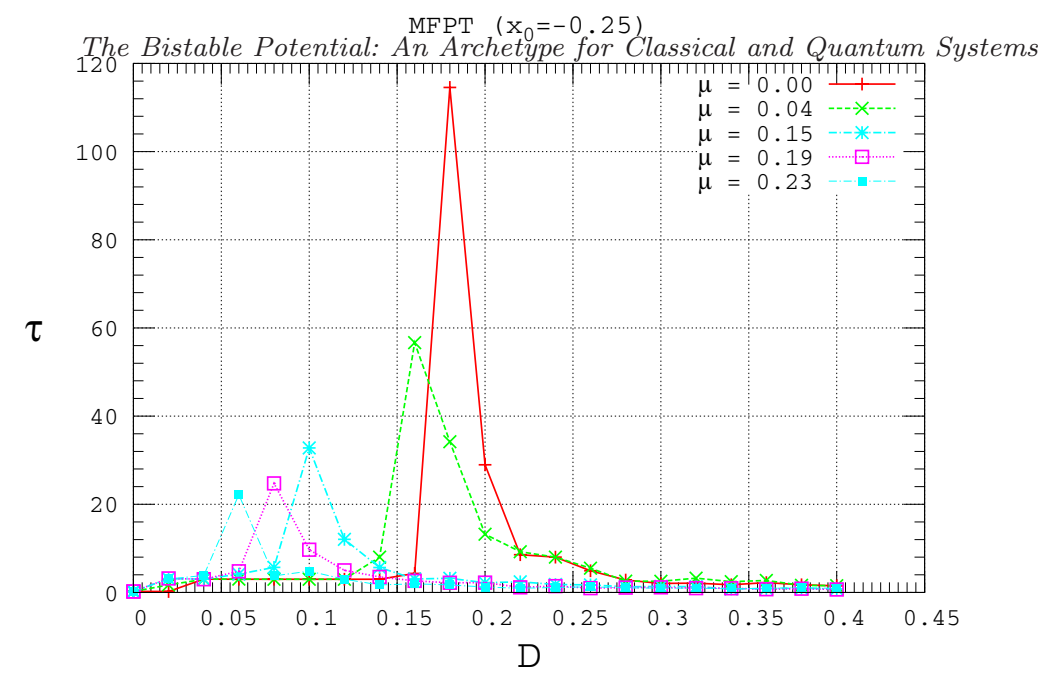

Fig. 2. (Color online) Plot of the MET as function of $D$ for five different values of the parameter $\mu$, namely $\mu=0.0,0.04,0.15,0.19,0.23$.

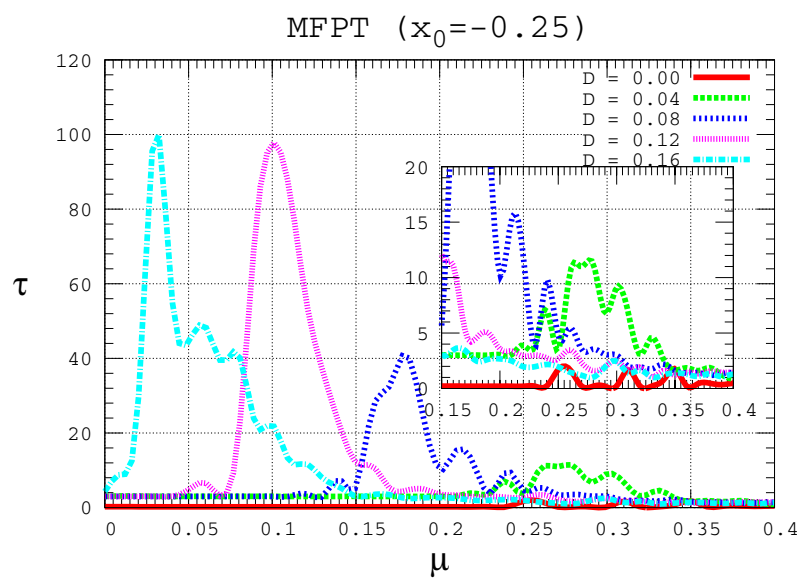

Fig. 3. (Color online) Plot of the behavior of the MFPT as function of $\mu$ for several values of the parameter $D$, namely $D=0.0$ (continuous red line), $D=0.04$ (dot green line), $D=0.08$ (dot blue line), $D=0.12$ (dot magenta line), $D=0.16$ (aqua dot line). Inset: magnification of the behaviors for low noise intensities.

\section{Lévy Noise in Population Dynamics}

The random walk of a particle subject to a Lévy noise source is characterized by "flights" appearing in the particle trajectory that show diffusion properties very different from those obtained in the presence of a Gaussian noise source. Lévy flights have been observed in many physical, biological and social systems, where scale-invariance phenomena take place ${ }^{8,23-25}$ (for a recent review on Lévy flights see Ref. 8 and references therein). Recently noise induced transitions, such as stochastic resonance, resonant activation and noise enhanced stability were observed in the 

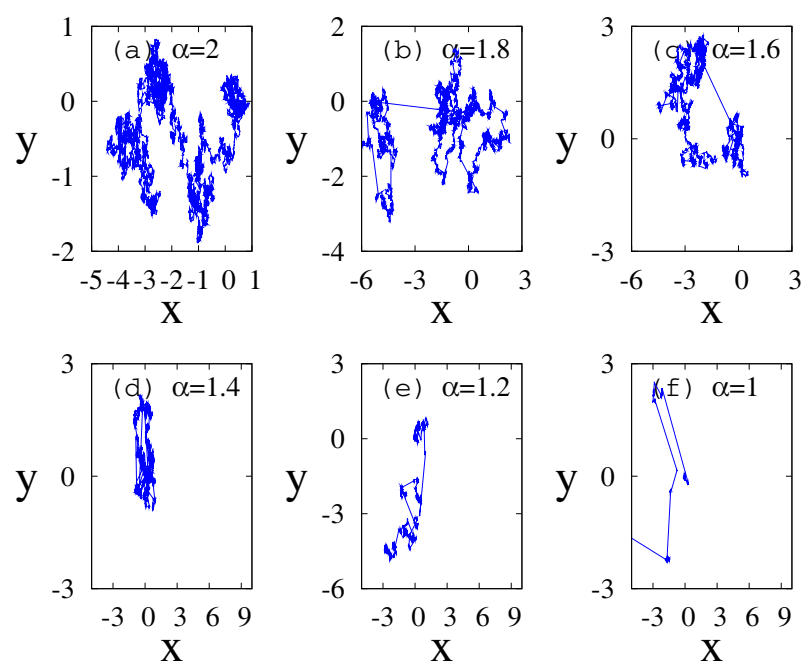

Fig. 4. (Color online) Two-dimensional trajectories of free diffusion of a particle subjected to noise sources with six different values of the shape parameters. Namely: (a) Gaussian $(\alpha=2, \beta=$ $0)$; (b)-(e) Lévy $(\alpha=1.8,1.6,1.4,1.2)$; (f) Cauchy-Lorentz $(\alpha=1, \beta=0)$. The values of the other parameters are $\mu=0, \sigma=1$ and $\beta=0$. Arbitrary units are used in both axes.

presence of Lévy noise. ${ }^{26-28}$ Moreover, Lévy flights have been successfully used to describe the spatial distributions of predators or foragers in ecological systems. ${ }^{29,30}$ Lévy flights are stochastic processes characterized by the occurrence of extremely long jumps, and their trajectories are not continuous. The length of these jumps is distributed according to a Lévy stable statistics with a power-law tail and divergence of the second moment. Such distributions form a four-parameter family of continuous probability distributions with location and scale parameters $\mu$ and $\sigma$, respectively, and two shape parameters $\beta$ (asymmetry parameter) and $\alpha$ (index of stability or characteristic exponent). ${ }^{31}$ In Fig. 4, sample trajectories for six different values of $\alpha$ and $\beta$ parameters are shown. We note that for $\beta=0$, a Lévy symmetric $\alpha$-stable distribution is obtained.

In this paper we investigate the stochastic dynamics of two competing species within the formalism of the generalized Lotka-Volterra equations in the presence of multiplicative Lévy noise sources

$$
\begin{aligned}
& \frac{d x}{d t}=m x(a-x-\gamma(t) y)+x \xi_{x}^{\alpha, \beta}(t), \\
& \frac{d y}{d t}=m y(a-y-\gamma(t) x)+y \xi_{y}^{\alpha, \beta}(t),
\end{aligned}
$$

where $a$ is the growth parameter and $\gamma(t)$ is the time-dependent interaction parameter between the species. Here $\xi_{x}^{\alpha, \beta}(t)$ and $\xi_{y}^{\alpha, \beta}(t)$ are statistically independent $\alpha$-stable Lévy noises with zero mean $(\mu=0)$. The noise intensity $D=\sigma^{\alpha}$ is assumed to be equal for the two noise sources. Here $\sigma$ is the scale parameter of the Lévy distribution. The time series for the two populations are obtained setting $m=70$ 
and $a=1$. It is known that for $\gamma<1$ a coexistence regime takes place, while for $\gamma>1$ an exclusion regime is established. ${ }^{32-34}$ Coexistence of the two species and exclusion of one of them correspond to stable states of the Lotka-Volterra's deterministic model. Because of the strong interaction between the ecological system and the environment, the interaction parameter $\gamma(t)$ is affected both by deterministic periodical "forces", e.g., temperature, and random fluctuations of environmental variables such as food resources. As a consequence, noise together with periodic forces determines the crossing from one dynamical regime $(\gamma<1 \rightarrow$ coexistence) to the other one $(\gamma>1 \rightarrow$ exclusion). This continuous and noisy behavior of the interaction parameter $\gamma(t)$ can be described by the stochastic differential equation

$$
\frac{d \gamma(t)}{d t}=-\frac{\partial U(\gamma, t)}{\partial \gamma}+\xi_{\gamma}^{\alpha, \beta}(t)
$$

where

$$
U(\gamma, t)=h(\gamma-1)^{4} / \eta^{4}-2 h(\gamma-1)^{2} / \eta^{2}+A \gamma \cos \left(\omega_{0} t\right)
$$

is the time-dependent bistable quartic potential, and $\xi_{\gamma}^{\alpha, \beta}(t)$ represents the random fluctuations of environmental variables.

The effect of the noise on the time behavior of the interaction parameter $\gamma(t)$ consists in causing jumps from the left-side well $(\gamma=0.5)$ to the right-side $(\gamma=$ 1.5) of the potential $U(\gamma, t)$. The alternating coexistence/exclusion regime can be modulated by the specific Lévy noise source $\xi_{\gamma}^{\alpha, \beta}(t)$ with $\mu=0$, varying both the intensity $D_{\gamma}$ and the parameters $\alpha$ and $\beta$. The synchronization effect observed in the presence of a suitable intensity of Gaussian noise is a well-known effect, named stochastic resonance (SR), ${ }^{35,36}$ and could affect considerably the dynamics of real ecosystems. One signature of the SR phenomenon is the presence of a maximum in the signal power amplification (SPA) $\eta$ as a function of the noise intensity. ${ }^{35}$ The SPA is defined as the ratio of the power of the output signal $\gamma(t)$ sampled at the frequency $\Omega$ of the external driving, to the power of the driving signal. In Fig. 5 we show the SPA behavior for symmetric $(\beta=0)$ Lévy noise sources and different values of the index $\alpha$ of the stable Lévy distribution. The nonmonotonic behavior of the SPA indicates clearly the presence of stochastic resonance, characterized by a maximum whose value decreases as the index $\alpha$ approaches 1 (Cauchy distribution). The behavior for $\alpha=2$ corresponds to the Gaussian noise source.

Now we analyze the dynamics of the two species densities for different symmetrical $(\beta=0) \alpha$-stable Lévy distributions. We consider the noise intensity $D_{\gamma}$ corresponding to the maximum of SPA in Fig. 5, and solve numerically Eqs. (4) and (5) for different values of the multiplicative noise intensity $D$. The alternating dynamical regime, resulting from the quasi-periodical oscillation of $\gamma(t)$ between the two potential wells, determines the anticorrelated oscillating behavior of the two species. Moreover, a multiplicative noise intensity exists that induces oscillating behavior with a maximum of anticorrelation between the two species breaking the symmetric dynamical behavior of the ecosystem. ${ }^{33}$ Therefore, we calculate the 
B. Spagnolo et al.

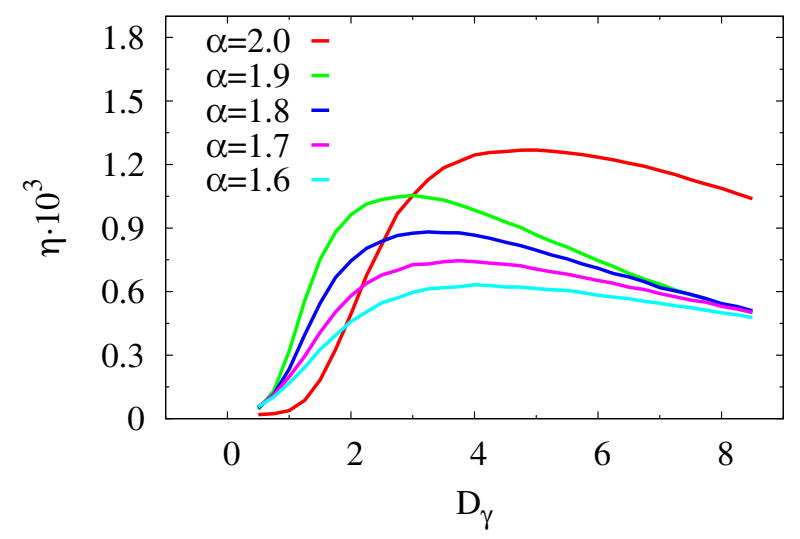

Fig. 5. (Color online) Spectral power amplification $\eta$ of $\gamma(t)$ as a function of the noise intensity $D_{\gamma}$, for different values of the index $\alpha$ of the stable Lévy distribution, namely $\alpha=1.6,1.7,1.8$, 1.9, 2.0 .

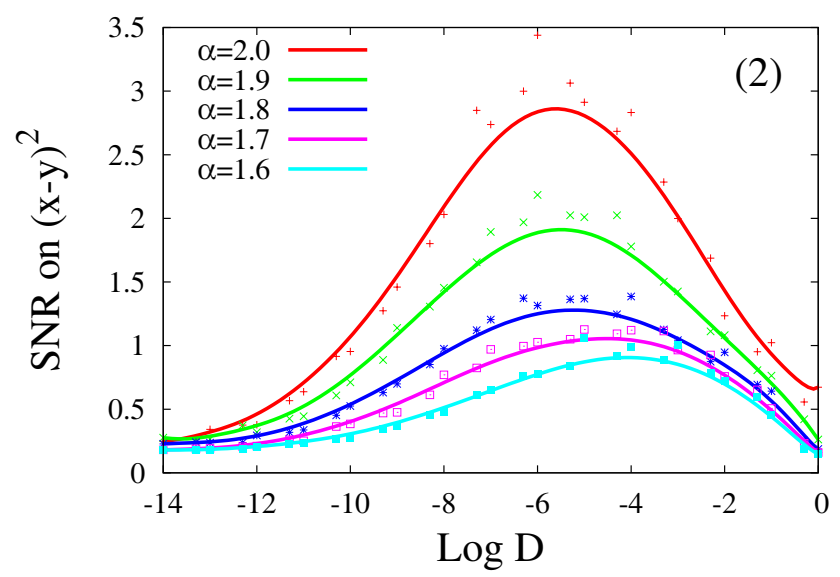

Fig. 6. (Color online) The SNR of the quantity $(x-y)^{2}$ as a function of the noise intensity $D$, for different values of the index $\alpha$, namely $\alpha=1.6,1.7,1.8,1.9,2.0$, and $\beta=0$. Here we set $D_{\gamma}$ at the value that maximizes the SPA curves of the parameter $\gamma(t)$. Red plus, green cross, blue star, pink empty square and light blue full square represent the SNR values calculated by numerical integration of Eqs. (4)-(6). The solid lines are the curves obtained by interpolating the numerical data. Each curve corresponds to the symbols with the same color.

signal-to-noise ratio (SNR) of $(x-y)^{2}$ for different values of $\alpha$. The results are shown in Fig. 6. We can observe the presence of a nonmonotonic behavior characterized by a maximum, which is the other signature of the SR phenomenon. This confirms that the multiplicative noise is responsible for a further SR phenomenon affecting directly the dynamics of the two species. The maximum of the SNR decreases as $\alpha$ approaches 1, according to the behavior of SPA observed for the interaction parameter $\gamma$ (see Fig. 5). 


\section{Quantum System}

In a dissipative quantum system interacting with a thermal bath, the quantum tunneling can play an important role on the relaxation time from a metastable state. ${ }^{9}$ During the last decades the effects of environment on quantum tunneling phenomenon have been intensively studied..$^{9,37-40}$ In this context, symmetric and asymmetric quantum bistable systems are good enough to analyze superconducting quantum bits and decoherence phenomena. ${ }^{41,42}$

\subsection{The model}

In order to analyze the evolution of a quantum particle subjected to timeindependent asymmetric bistable potential and affected by environmental noise, we use the Caldeira-Leggett model. ${ }^{37}$ The study is performed by using the approach of the Feynman-Vernon functional ${ }^{43}$ in discrete variable representation (DVR). ${ }^{44}$ Our physical model consists of a quantum particle with mass $M$, interacting with a thermal bath which plays the role of environment. The unperturbed Hamiltonian of the system, where $\hat{q}$ and $\hat{p}$ are one-dimensional operators for position and momentum respectively, is

$$
\hat{H}_{0}=\frac{\hat{p}^{2}}{2 M}+\hat{V}_{0}(\hat{q})
$$

where

$$
\hat{V}_{0}(\hat{q})=\frac{M^{2} \omega_{0}^{4}}{64 \Delta U} \hat{q}^{4}-\frac{M \omega_{0}^{4}}{4} \hat{q}^{2}-\hat{q} \epsilon,
$$

is the asymmetric bistable potential shown in Fig. 7. Here, $\epsilon$ and $\Delta U$ are the asymmetry parameter and the barrier height, respectively, and $\omega_{0}$ is the natural oscillation frequency. Here we study the dynamics of the system using this specific shape of potential, considering only the eight lowest energy eigenstates, whose eigenvalues are shown on the vertical axis of Fig. 7. This allows the quantum particle to escape from the metastable state only via the tunnel effect. In the same figure, on the horizontal axis the eight position eigenvalues are displayed. The dark blue rectangle marks the initial position of the particle. Specifically, this position does not coincide with any energy eigenstate, and therefore is given by a proper linear combination of the eight energy eigenstates. The curves shown in the figures are the eigenfunctions corresponding to the eight energy eigenvalues. The Hamiltonian can be split as follows

$$
\hat{H}=\hat{H}_{0}+\hat{H}_{B}
$$

where

$$
\hat{H}_{B}=\sum_{j=1}^{\mathcal{N}} \frac{1}{2}\left[\frac{\hat{p}_{j}^{2}}{m_{j}}+m_{j} \omega_{j}^{2}\left(\hat{x}_{j}-\frac{c_{j}}{m_{j} \omega_{j}^{2}} \hat{q}\right)^{2}\right]
$$




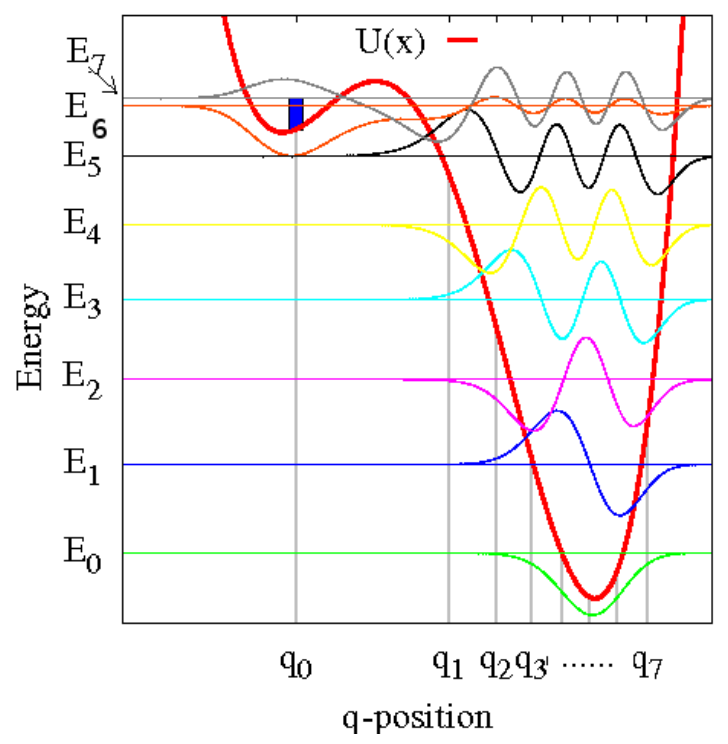

Fig. 7. (Color online) Potential profile $V_{0}(q)$ [see Eq. (9)] for $\Delta U=3$ and $\epsilon=0.6$. Energy levels and corresponding eigenstates considered in our analysis are indicated by horizontal lines and curves, respectively. The initial position is $q_{\text {start }}=q_{0}$ (blue rectangle).

is the Hamiltonian which describes the thermal reservoir and its interaction with the particle, according to the Caldeira-Leggett model. The thermal bath consists of an ensemble of $\mathcal{N}$ harmonic oscillators with spatial coordinate $\hat{x}_{j}$, momentum $\hat{p}_{j}$, mass $m_{j}$, and frequency $\omega_{j}$. The coefficients $c_{j}$ are the coupling constants between system and thermal bath. If $\mathcal{N} \rightarrow \infty$, we have a continuous spectral density. In the Markovian limit, which corresponds to the dissipation in the Ohmic regime, the damping is frequency independent, i.e., $\gamma(\omega)=\gamma$, and the spectral density is given by ${ }^{37} J(\omega)=\eta \omega$, where $\eta$ is the coupling strength between the system and the thermal bath, and represents the intensity of the environmental noise. In our model, we choose a spectral density characterized by an exponential cut-off with a cutting frequency $\omega_{c}$ as follows

$$
J(\omega)=\eta \omega \exp \left(-\frac{\omega}{\omega_{c}}\right) .
$$

\subsection{The Feynman-Vernon approach}

Following the Feynman-Vernon approach we write the density operator as follows

$$
\rho\left(q_{f}, q_{f}^{\prime} ; t\right)=\int d q_{0} \int d q_{0}^{\prime} K\left(q_{f}, q_{f}^{\prime}, t ; q_{0}, q_{0}^{\prime}, t_{0}\right) \rho_{S}\left(q_{0}, q_{0}^{\prime}, t_{0}\right),
$$

where the propagator $K$ is given by

$$
K\left(q_{f}, q_{f}^{\prime}, t ; q_{0}, q_{0}^{\prime}, t_{0}\right)=\int_{q\left(t_{0}\right)=q_{0}}^{q(t)=q_{f}} \mathcal{D} q \int_{q^{\prime}\left(t_{0}\right)=q_{0}^{\prime}}^{q^{\prime}(t)=q_{f}^{\prime}} \mathcal{D} q^{\prime} \mathcal{A}[q] \mathcal{A}^{*}\left[q^{\prime}\right] \mathcal{F}_{\mathrm{FV}}\left[q, q^{\prime}\right]
$$


and

$$
\mathcal{A}[q]=\exp \left(i \frac{S_{S}[q]}{\hbar}\right)
$$

with $S_{S}[q]$ being the classical action functional. In Eq. (14), $\mathcal{F}_{\mathrm{FV}}\left[q, q^{\prime}\right]=$ $\exp \left(-\left(\phi_{\mathrm{FV}}\left[q, q^{\prime}\right] / \hbar\right)\right)$ is the Feynman-Vernon (FV) influence functional with the influence weight functional $\phi_{\mathrm{FV}}\left[q, q^{\prime}\right]$, which is depending on the bath correlation function. ${ }^{40}$

\subsection{Discrete variable representation}

By solving the eigenvalue equation connected with the Hamiltonian $\hat{H}_{0}$ we get the energy eigenstates. If we choose to change the basis of the eigenstates going from the energy representation to the position one, we can describe the localization of the quantum particle. We recall that the eigenvalues of the position operator $\hat{q}$ in the basis $\left\{\left|q_{\mu}\right\rangle\right\}$ are shown in the horizontal axis of Fig. 7 .

Within the framework of the discrete variable representation (DVR) ${ }^{44}$ the dynamics is described by the quantum mechanical paths $q(t)$, which the system follow during its evolution in time. The system starts at time $t=t_{0}$ in the state $q\left(t_{0}\right)=q_{0}$ and evolves via $m$ jumps between the $M$ discrete states into the final state $q\left(t_{m}\right)=q_{m}$. The double path integral over the $m$-state paths $q(t)$ and $q^{\prime}(t)$ in Eq. (13) is rewritten as an integral over a single path that jumps between the $M^{2}$ states of the reduced density matrix in the $\left(q, q^{\prime}\right)$-plane. The total number $m$ of jumps is given by the sum of the number of jumps for the paths $q$ and $q^{\prime}$. The path can be split in two kinds of subpaths in the relative $(\xi(t))$ and center of mass $(\chi(t))$ coordinates as follows

$$
q(t)=\left[q(t)-q\left(t^{\prime}\right)\right]+\left[q\left(t^{\prime}\right)+q(t)\right]=[\xi(t)]+[\chi(t)]
$$

The system may be in a state where $\xi(t)=0$ and $\chi(t) \neq 0$ or a state where $\xi(t) \neq 0$ and $\chi(t) \neq 0$. The first kind of states is called sojourn and the second kind of states is called blip. From the previous definition it is clear that the sojourns are the diagonal states of the density matrix in the DVR representation while the blip are the off-diagonal states.

\subsection{Master equation}

Applying the Non-Interacting Cluster Approximation ${ }^{9}$ or NICA it is possible to obtain the following master equation (ME)

$$
\dot{\rho}_{\mu \mu}(t)=\sum_{\nu=1}^{N} \int_{t_{0}}^{t} d t^{\prime} \mathcal{H}_{\mu \nu}\left(t-t^{\prime}\right) \rho_{\nu \nu}\left(t^{\prime}\right),
$$


where $\mu=1, \ldots, N$ and $N$ is the number of eigenstates. The kernel of the Eq. (17) has a huge expression in terms of integrals calculated on all the paths as follows

$$
\begin{aligned}
\mathcal{H}_{\mu \nu}\left(t-t^{\prime}\right)= & \sum_{m=1}^{\infty} \int_{t_{0}}^{t} \mathcal{D}\left[t_{j}\right] \sum_{\mu_{j} \nu_{j}} \exp \left\{i \sum_{j=0}^{m-1} \int_{t_{j}}^{t_{j+1}} d t^{\prime} \times\left[E_{\mu_{j}}\left(t^{\prime}\right)-E_{\nu_{j}}\left(t^{\prime}\right)\right]\right\} \\
& \times \prod_{j=0}^{m-1}(-1)^{\delta_{j}}\left(\frac{i}{2}\right)^{m} \Delta_{j} \\
& \times \exp \left\{\sum_{l=1}^{m} \sum_{j=0}^{l-1} \xi_{l} S\left(t_{l}-t_{j}\right) \xi_{j}+i \sum_{l=1}^{m} \sum_{j=0}^{l-1} \xi_{l} R\left(t_{l}-t_{j}\right) \chi_{j}\right\},
\end{aligned}
$$

and therefore takes into account all possible transitions in the DVR paths. ${ }^{9}$ Using the NICA approximation we have the approximated ME

$$
\dot{\rho}_{\mu \mu}(t)=\sum_{\nu=1}^{N} \Gamma_{\mu \nu}(t) \rho_{\nu \nu}(t) .
$$

The rate which determines the dynamics over the largest time scale is the quantum relaxation rate

$$
\Gamma \equiv \min \left\{\left|\Re\left(\Lambda_{\nu}\right)\right| ; \nu=2, \ldots, N\right\},
$$

where $\Lambda_{\nu}$ are the eigenvalues of the rate matrix and $\left|\Re\left(\Lambda_{\nu}\right)\right|$ are the nonzero absolute values of the real part of $\Lambda_{\nu}$. We focus the analysis on the medium-short time behavior of the system, using the largest $\Lambda_{\nu}^{-1}$ as time scale to analyze the non-equilibrium dynamics of the quantum particle in the presence of thermal fluctuations.

\subsection{Results}

In this section we study the time evolution of our quantum particle taking into account the eight energy levels shown in Fig. 7. The analysis is restricted to the eight lowest levels of the system in order to point out the contribution of the tunnel effect to the dynamics of the particle. We analyze the time behavior of the populations for different values of the coupling strength, focusing on the time behavior of the state $\left|q_{0}\right\rangle$ (left well of the potential) choosing in the DVR-representation the position $q_{\text {start }}=q_{0}$. By integrating Eq. (17) for different values of the parameter $\eta$, the eigenstates $\left|q_{\mu}\right\rangle$ are considered and the time behavior of the corresponding population $\rho_{q_{\mu}} \equiv \rho_{\mu \mu}$ is obtained. Then, by a simple change of basis, it is possible to calculate the time evolution of the populations in the energy representation. It is worth noting that for each value of the noise intensity $\eta$ there are $N$ relaxation times. In order to set the time scale to observe the transient behavior, it is convenient to consider the maximum of these relaxation times. Simulations show that this time increases rapidly for larger values of $\eta$. Therefore, to describe the 


\section{at time $\tau / 4$}
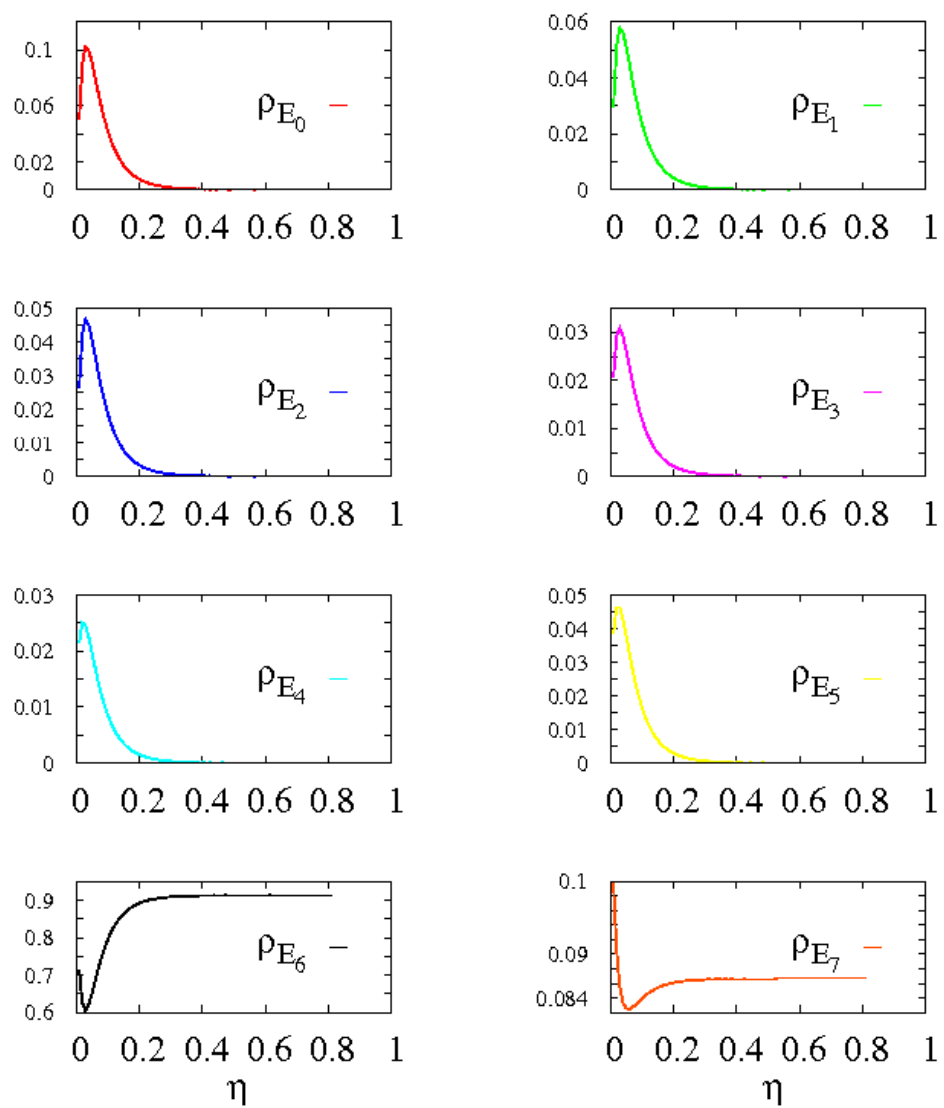

Fig. 8. (Color online) Population of the energy levels as a function of the noise intensity $\eta$ for $t=\tau / 4$.

time evolution of the system for different values of $\eta$, we choose as time scale $\tau$ the largest of the relaxation times obtained for $\eta=0.01$. This choice allows to follow the transient dynamics of the system for low and intermediate values of the coupling constant. In Figs. 8 and 9 we show the behavior of the populations of the eight energy levels as a function of $\eta$ for two different times.

Our initial condition (see Fig. 7) implies that the only energy eigenfunctions involved in the initial state are $\left|E_{6}\right\rangle$ and $\left|E_{7}\right\rangle$. In particular, as one can see in Figs. 8 and 9 , the greater contribution is due to the eigenstate $\left|E_{6}\right\rangle$. Moreover, we note that the eigenfunctions $\left|E_{6}\right\rangle$ and $\left|E_{7}\right\rangle$ are the only ones that do not vanish in the left well, and so we can think of them as the "left" eigenstates, considering the other ones as the "right" eigenstates. We can see that for $t=\tau / 4$ we have, for all the energy levels, a nonmonotonic behavior of the populations as a function of the noise intensity, with an extremum at $\eta \approx 0.05$. For the eigenstates $\left|E_{6}\right\rangle$ and $\left|E_{7}\right\rangle$ 
at time $6 \tau$
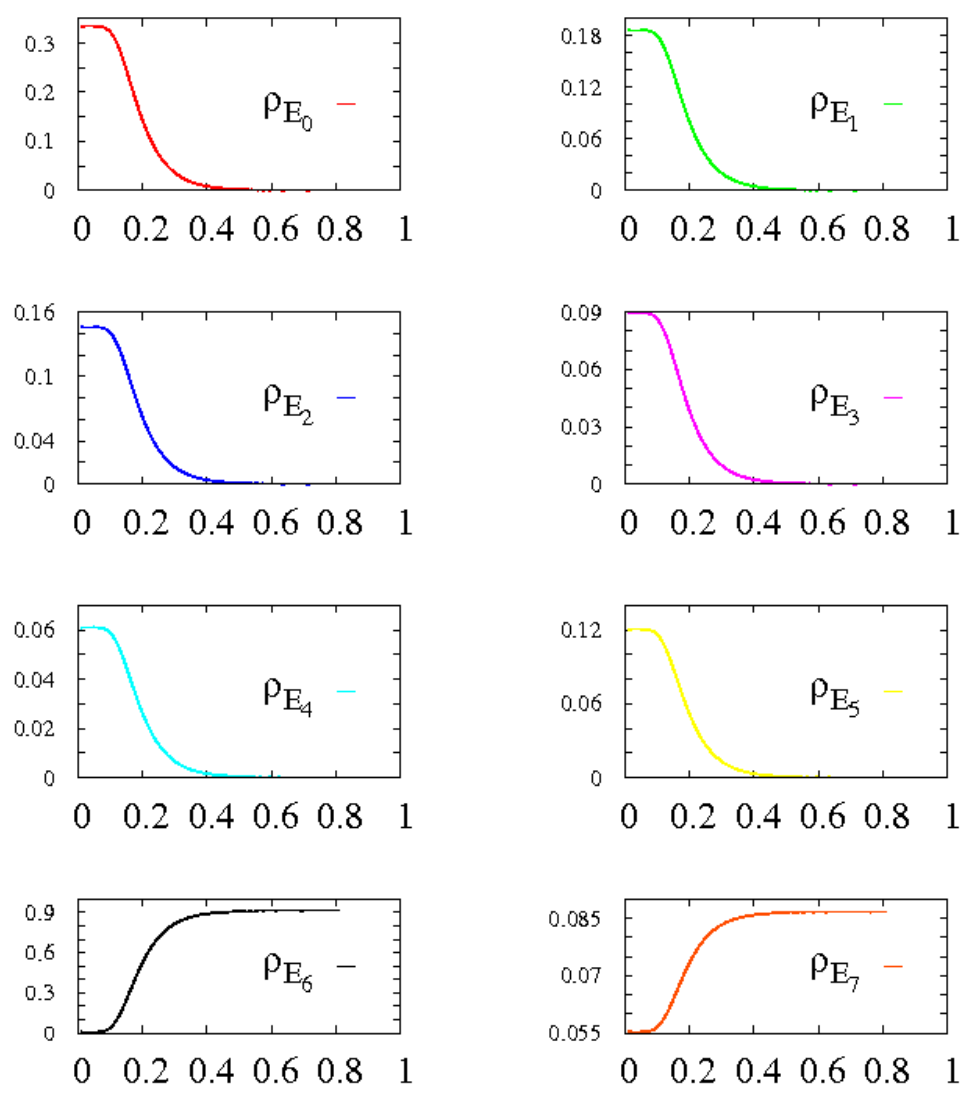

$\eta$

$\eta$

Fig. 9. (Color online) Population of the energy levels as a function of the noise intensity $\eta$ for $t=6 \tau$.

in particular we note a minimum, while for the "right" eigenstates we observe a maximum. This can be interpreted as an escape of the particle from the left well (the metastable one) toward the right one (the stable one). For $\eta>0.2$ we see that the populations tend to constant values both for $t=\tau / 4$ and $t=6 \tau$. This behavior can be explained by the quantum Zeno effect, responsible for a slowing down or, for higher values of the noise intensity $\eta$, a freezing of the dynamics. ${ }^{45}$

\section{Acknowledgments}

We acknowledge the support of Ministero dell'Istruzione, dell'Università e della Ricerca (Italian Government) and the Russian Foundation for Basic Research (project 11-02-01418). This work was also partially supported by the EU through Grant No. PITN-GA-2009-234970. 


\section{References}

1. J. D. Gunton and M. Droz, Introduction to the Theory of Metastable and Unstable States (Springer, Berlin, 1983).

2. A. Strumia and N. Tetradis, J. High Energy Phys. 11, 023 (1999).

3. P. G. Debenedetti and F. H. Stillinger, Nature 410, 267 (2001).

4. B. Spagnolo et al., Acta Phys. Pol. B 38, 1925 (2007).

5. N. Agudov and B. Spagnolo, Phys. Rev. E 64, 035102(R) (2001).

6. A. A. Dubkov and B. Spagnolo, Phys. Rev. E 69, 061103 (2004).

7. A. Fiasconaro, J. J. Mazo and B. Spagnolo, Phys. Rev. E 82, 041120 (2010).

8. A. A. Dubkov, B. Spagnolo and V. V. Uchaikin, Int. J. Bifurc. Chaos 18, 2649 (2008).

9. M. Thorwart, M. Grifoni and P. Hänggi, Ann. Phys. 293, 15 (2001).

10. W. Horsthemke and R. Lefever, Noise-Induced Transitions (Springer, Berlin, 1984).

11. C. Van den Broeck, J. M. R. Parrondo and R. Toral, Phys. Rev. Lett. 73, 3395 (1994).

12. M. A. Muñoz, F. Colaiori and C. Castellano, Phys. Rev. E 72, 056102 (2005).

13. A. Manor and N. M. Shnerb, Phys. Rev. Lett. 103, 030601 (2009).

14. O. Chichigina, D. Valenti and B. Spagnolo, Fluct. Noise Lett. 5, L243 (2005).

15. F. de Pasquale et al., Phys. Rev. A 33, 4360 (1986).

16. B. Y. Zhu, F. Marchesoni and F. Nori, Phys. Rev. Lett. 92, 180602 (2004).

17. P. I. Hurtado, J. Marro and P. L. Garrido, J. Stat. Phys. 133, 29 (2008).

18. A. Fiasconaro, D. Valenti and B. Spagnolo, Physica A 325, 136 (2003).

19. A. Fiasconaro and B. Spagnolo, Phys. Rev. E 80, 041110 (2009).

20. V. I. Klyatskin, Stochastic Equations Through the Eye of the Physicist (Elsevier Science, Oxford, 2005).

21. R. L. Stratonovich, Introduction to the Theory of Random Noise (Gordon and Breach, New York, 1963).

22. C. W. Gardiner, Handbook of Stochastic Methods (Springer Verlag, Berlin and Heidelberg, 1985).

23. A. V. Chechkin et al., Adv. Chem. Phys. 133, 439 (2006).

24. B. Dybiec, E. Gudowska-Nowak and I. M. Sokolov, Phys. Rev. E 76, 041122 (2007).

25. A. A. Dubkov and B. Spagnolo, Eur. Phys. J. B 65, 361 (2008).

26. B. Dybiec and E. Gudowska-Nowak, New J. Phys. 9, 452 (2007).

27. B. Dybiec, Phys. Rev. E 80, 041111 (2009).

28. G. Augello, D. Valenti and B. Spagnolo, Eur. Phys. J. B 78, 225 (2010).

29. J. Bascompte and R. Sole, Modeling Spatiotemporal Dynamics in Ecology (Springer, Berlin, 1998).

30. S. Bertrand et al., J. Mar. Sci. 62, 477 (2005).

31. A. La Cognata et al., Phys. Rev. E 82, 011121 (2010).

32. J. M. G. Vilar and R. V. Solé, Phys. Rev. Lett. 80, 4099 (1998).

33. D. Valenti, A. Fiasconaro and B. Spagnolo, Physica A 331, 477 (2004).

34. D. Valenti et al., Eur. Phys. J. B 50, 199 (2006).

35. L. Gammaitoni et al., Rev. Mod. Phys. 70, 223 (1998).

36. R. N. Mantegna, B. Spagnolo and M. Trapanese, Phys. Rev. E 63, 011101 (2001).

37. A. O. Caldeira and A. L. Leggett, Phys. Rev. Lett. 46, 211 (1981).

38. A. J. Leggett et al., Rev. Mod. Phys. 59, 1 (1987).

39. M. Grifoni and P. Hänggi, Phys. Rep. 304, 229 (1998).

40. U. Weiss, Quantum Dissipative Systems (World Scientific, Singapore, 1999).

41. J. R. Friedman et al., Nature 406, 43 (2000). 
B. Spagnolo et al.

42. J. M. Martinis et al., Phys. Rev. Lett. 89, 117901 (2002).

43. R. P. Feynman and F. L. Vernon, Jr., Ann. Phys. 24, 118 (1963).

44. D. O. Harris, G. G. Engerholm and W. D. Gwinn, J. Chem. Phys. 43, 1515 (1965).

45. P. Facchi et al., Phys. Rev. A 71, 022302 (2005). 